\title{
On Skew E-W Matrices
}

\author{
José Andrés Armario and María Dolores Frau
}

Departamento de Matemática Aplicada I, Universidad de Sevilla, Sevilla, Spain, E-mail: armario@us.es,mdfrau@us.es

\begin{abstract}
An E-W matrix $M$ is a $(-1,1)$-matrix of order $4 t+2$, where $t$ is a positive integer, satisfying that the absolute value of its determinant attains Ehlich-Wojtas' bound. $M$ is said to be of skew type (or simply skew) if $M-I$ is skew-symmetric where $I$ is the identity matrix. In this paper, we draw a parallel between skew E-W matrices and skew Hadamard matrices concerning a question about the maximal determinant. As a consequence, a problem posted on Cameron's website [7] has been partially solved. Finally, codes constructed from skew E-W matrices are presented. A necessary and sufficient condition for these codes to be self-dual is given, and examples are provided for lengths up to 52.
\end{abstract}

Keywords: skew E-W matrices; maximal determinants; self-dual codes

\section{INTRODUCTION}

Throughout this paper, for convenience, when we say determinant of a matrix we mean the absolute value of the determinant. Hadamard showed in [15] that $n^{n / 2}$ was a bound for the determinant of an $n$ by $n$ matrix with entries \pm 1 . This bound can be attained only if $n$ is a multiple of 4. A matrix that attains it is called a Hadamard matrix, and it is an outstanding conjecture that one exists for any multiple of 4 . For other orders more stringent bounds have been established.

Let $g(n)$ denote the maximum determinant of all $n \times n$ matrices with elements \pm 1 . When $n \equiv 2 \bmod 4$, Ehlich [9] and independently Wojtas [26] proved that

$$
g(n) \leq(2 n-2)(n-2)^{n-1}(\text { Ehlich--Wojtas' bound }) .
$$

This bound can be attained only if $2 n-2$ is the sum of two squares. A matrix that attains it is called an $E-W$ matrix. It has been conjectured that $\mathrm{E}-\mathrm{W}$ matrices of order $n$ exist 
TABLE I. Determinants bounds. The last bound is given in Theorem 2.3.

\begin{tabular}{ccccc}
\hline & $g(n)$ & $f(n)$ & $g_{k}(n)$ & $f_{k}(n)$ \\
\hline$n \equiv 0 \bmod 4$ & $n^{n / 2}$ & $(n-1)^{n / 2}$ & $n^{n / 2}$ & $(n-1)^{n / 2}$ \\
$n \equiv 2 \bmod 4$ & $(2 n-2)(n-2)^{\frac{1}{2} n-1}$ & $(n-1)^{n / 2}$ & $(2 n-2)(n-2)^{\frac{1}{2} n-1}$ & $(\mathbf{2 n}-\mathbf{3})(\mathbf{n}-\mathbf{3})^{\frac{1}{2} \mathbf{n}-\mathbf{1}}$
\end{tabular}

when $2 n-2=\alpha^{2}+\beta^{2}$ for some positive integers $\alpha$ and $\beta$. The interested reader is addressed to [20] and the website [24] for further information on what is known about maximal determinants.

There is a companion theory for matrices with 0's on the main diagonal and \pm 1 elsewhere. Let $f(n)$ denote the maximum determinant of all $n \times n$ matrices with elements 0 on the main diagonal and \pm 1 elsewhere. It is well-known that $f(n) \leq(n-1)^{n / 2}$. This can be attained only when $n$ is even, and a matrix $C$ that does so is called a conference matrix. For $n$ odd the question has been hardly worked out [6].

The study of the maximum determinant of matrices with additional structural properties is also an interesting problem. For instance, let $\mathcal{F}_{k}(n)$ be the set of all $n \times n$ skewsymmetric matrices with elements 0 on the main diagonal and \pm 1 elsewhere and $f_{k}(n)$ will denote the maximum determinant of the matrices in $\mathcal{F}_{k}(n)$. Respectively, let $\mathcal{G}_{k}(n)$ be the set of all $(-1,1)$-matrices of skew type of order $n$ (i.e. $(-1,1)$-matrices of the form $M+I$ where $\left.M \in \mathcal{F}_{k}(n)\right)$ and $g_{k}(n)$ will denote the maximum determinant of the matrices in $\mathcal{G}_{k}(n)$. In [7], $f_{k}(n)$ and $g_{k}(n)$ has been asked for different values of $n$.

Remark 1. For $n$ even, the attainable upper bounds for $g(n)$ (Hadamard's bound when $n \equiv 0 \bmod 4$ and Ehlich-Wotjas' bound when $n \equiv 2 \bmod 4)$ collapse in only one bound for $f(n)$ (see columns 1 and 2 in Table I). It seems that Ehlich-Wojtas' bound does not have an analogous one in $f(n)$.

Two matrices $M$ and $N$ are said to be Hadamard equivalent or equivalent if one can be obtained from the other by a sequence of the operations:

- interchange any pairs of rows and/or columns;

- multiply any rows and/or columns through by -1 .

In other words, we say that $M$ and $N$ are equivalent if there exist $(0,1,-1)$-monomial matrices $P$ and $Q$ such that $P M Q^{T}=N$.

A matrix $M$ is symmetric if $M=M^{T}$. A matrix is skew-symmetric (or skew) if $M=$ $-M^{T}$. The following theorem analyzes the structure of the conference matrices [19], p. 307]:

Theorem 1.1. If $C$ is an $n \times n$ conference matrix, then either $n \equiv 0 \bmod 4$ and $C$ is equivalent to a skew matrix, or $n \equiv 2 \bmod 4$ and $C$ is equivalent to a symmetric matrix. In the later case, $n-1$ is the sum of two squares: thus they cannot exist for orders 22, 34, $58,70,78,94$. The first values for which the existence of symmetric conference matrices is unknown are $n=66,86$.

It is natural to ask whether there is any relationship between $g(n)$ and $f(n)$. Concretely, if $\operatorname{det}(M)=f(n)$ then $\operatorname{det}(M+I)=g(n)$ ? 
Assuming that the matrices are skew, the same question has been stated in the problem 104 posted on Cameron's website (see [7]). This problem arose in the context of experimental designs [25] and it was suggested by Dennis Lin. Concretely, it asks to decide whether or not the following equivalence holds:

$M$ has maximum determinant among the matrices in $\mathcal{F}_{k}(n)$ if and only if $M+I$ has maximum determinant among the matrices in $\mathcal{G}_{k}(n)$.

First we deal with the more general situation, and in the next section we will address Cameron's problem.

It is known that if $C$ is a skew conference matrix then $H=C+I$ is a (skew) Hadamard matrix and vice versa. Therefore, for $n \equiv 0 \bmod 4$

$$
f(n)=(n-1)^{n / 2} \Longrightarrow g(n)=n^{n / 2},
$$

and conversely, if a skew Hadamard exists then,

$$
g(n)=n^{n / 2} \Longrightarrow f(n)=(n-1)^{n / 2} .
$$

It is conjectured that $f(n)=(n-1)^{n / 2}$ for all order multiple of 4 . The first open order is $n=276$. Let us point out that there are Hadamard matrices of order $n=276$ but a skew Hadamard matrix is unknown.

Skew conference and skew Hadamard matrices are of great interest because of their elegant structure, their beautiful properties and their applications to Coding Theory, Combinatorial Designs, and Cryptography [12, 18, 21, 25].

The situation for $n \equiv 2 \bmod 4$ is quite different. Let $C_{6}$ be the (symmetric) conference matrix of order 6 ,

$$
C_{6}=\left[\begin{array}{cccccc}
0 & 1 & 1 & 1 & 1 & 1 \\
1 & 0 & 1 & 1 & - & - \\
1 & 1 & 0 & - & - & 1 \\
1 & 1 & - & 0 & 1 & - \\
1 & - & - & 1 & 0 & 1 \\
1 & - & 1 & - & 1 & 0
\end{array}\right]
$$

consequently, $\operatorname{det}\left(C_{6}\right)=f(6)$ but $\operatorname{det}\left(C_{6}+I\right)=64<g(6)=160$. Furthermore, we found a skew-symmetric matrix of order 6

$$
S_{6}=\left[\begin{array}{cccccc}
0 & 1 & - & 1 & 1 & 1 \\
- & 0 & 1 & 1 & 1 & 1 \\
1 & - & 0 & 1 & 1 & 1 \\
- & - & - & 0 & - & 1 \\
- & - & - & 1 & 0 & - \\
- & - & - & - & 1 & 0
\end{array}\right]
$$

where $\operatorname{det}\left(S_{6}\right)<f(6) \operatorname{but} \operatorname{det}\left(S_{6}+I\right)=g(6)=160$. There is no regularity for $f(n)$ and $g(n)$ by means of the morphism $M \rightarrow M+I$.

In this paper, considering the subset of matrices $\mathcal{F}_{k}(n)$, we will recover Ehlich-Wotjas' bound for the determinant of these matrices (see Table I) and the relationship between $f(n)$ and $g(n)$ will be clarified in this extremal case. As a consequence, we will solve 
partially Cameron's problem. Finally, an application of skew E-W matrices to Coding Theory will be given.

Notation. Throughout the paper, we will use - for -1 and 1 for +1 . The notation $J_{n}$ stands for the matrix all whose entries are equal to one of order $n$ and $M^{T}$ for the transpose of $M$. Whenever a skew $(-1,1)$-matrix is mentioned in this paper, we mean a $(-1,1)$-matrix of skew type.

\section{SKEW E-W MATRICES}

This section is mainly devoted to analyzing the relationship between $g_{k}(n)$ and $f_{k}(n)$. In this study, we will show that skew E-W matrices arise as the analogous ones to skew Hadamard matrices when $n \equiv 2 \bmod 4$. Finally, we will outline a technique for finding such skew E-W matrices. It is worth mentioning that in [4], skew E-W matrices are characterized in terms of tournaments. This work provides another direction to study tournaments.

\subsection{Skew E-W Matrices and the Relationship between $f_{k}(n)$ and $g_{k}(n)$}

First, we recall a result for the determinant of skew symmetric matrices:

\section{Lemma 2.1. [16]}

1. If $n$ is odd and $A$ is a skew matrix with real elements the $\operatorname{det}(A)=0$.

2. If $n$ is even and $A$ a skew matrix with real elements then $\operatorname{det}(A)$ is $\operatorname{PF}(A)^{2}$, where $P F(A)$ is the Pfaffian of $A$ a polynomial in the entries of $A$.

For $n$ odd, $f_{k}(n)=0$ by Lemma 2.1; so there is not much to say. The case $n \equiv 0 \bmod 4$ has already been discussed in this manuscript. We shall here be concerned with the case $n \equiv 2 \bmod 4, n \neq 2$ and this will be implicitly assumed in what follows.

Firstly, we will indicate an upper bound for $f_{k}(n)$, before a technical result is needed.

Lemma 2.2. [8] Let $h \geq 2, A=\left[a_{i, j}\right]$ denote an $h$ by $h$ positive-definite symmetric matrix with diagonal elements $a_{i, i}=n-1$ and with $\left|a_{i, j}\right| \geq 2$. Then

1. $\operatorname{det}(A) \leq(n+2 h-3)(n-3)^{h-1}$.

2. equality holds if and only if $A=\Sigma L \Sigma$ for some suitable $\Sigma$, where $\Sigma$ is a square diagonal matrix with all its diagonal elements \pm 1 and $L=(n-3) I+2 J$.

Although the proof of the following result follows from a careful reading of the proof of [8, Theorem 1]. The given bound for $f_{k}(n)$ seems to be new and it corresponds with Ehlich-Wojtas' bound.

Theorem 2.3. We have

$$
f_{k}(n) \leq(2 n-3)(n-3)^{\frac{1}{2} n-1}
$$

and equality holds if and only if there exist $M \in \mathcal{F}_{k}(n)$ with

$$
M M^{T}=M^{T} M=\left[\begin{array}{ll}
L & 0 \\
0 & L
\end{array}\right],
$$

where $L=(n-3) I+2 J$. 
Proof. We have included a sketch of the proof to make the paper as much self-contained as possible.

If $M M^{T}=M^{T} M$ is of the form stated, then indeed holds the desired result, by Lemma 2.2.

Now suppose that $M \in \mathcal{F}_{k}$. Let $A=M M^{T}$. If no element of $A$ were to vanish, then by Lemma 2.2 we should have

$$
\left(f_{k}(n)\right)^{2}=\operatorname{det}(A)<(2 n-3)^{2}(n-3)^{n-2},
$$

and the result would follow.

Otherwise, at least one element of $A$ vanishes and in particular is divisible by 4 . Now for any $i, j, t$ three distinct integers all in the range 1 to $n$ inclusive,

$$
a_{i, i}+a_{i, j}+a_{j, t}+a_{t, i}=3 \bmod 4
$$

and so since $a_{i, i}=n-1=1 \bmod 4$ we find that

$$
a_{i, j}+a_{j, t}+a_{t, i}=2 \bmod 4
$$

By interchanging rows of $M$ if necessary, we can arrange to get a partition $A$ in the form

$$
A=\left[\begin{array}{cc}
B(n-h, n-h) & C(n-h, h) \\
D(h, n-h) & E(h, h)
\end{array}\right] .
$$

It then follows without difficulty from (3) that every off-diagonal element of $B$ and of $E$ is congruent to 2 modulo 4 , whereas every element of $C$ and $D$ is divisible by 4 . By Fischer's inequality and by Lemma 2.2,

$$
\left(f_{k}(n)\right)^{2}=\operatorname{det}(A) \leq(n-3)^{n-2}(2 n-3)^{2},
$$

with equality if and only if $h=\frac{1}{2} n$. Thus we have proved the inequality of the Theorem, and using Lemma 2.2, can have equality only if

$$
A=\left[\begin{array}{cc}
\Sigma_{1} L \Sigma_{1} & 0 \\
0 & \Sigma_{2} L \Sigma_{2}
\end{array}\right] .
$$

Now replacing $M$ by $\Sigma M \Sigma$ where

$$
\Sigma=\left[\begin{array}{cc}
\Sigma_{1} & 0 \\
0 & \Sigma_{2}
\end{array}\right]
$$

changes $M$ to a skew matrix in the form required. Obviously, $M^{T} M=M M^{T}$. This concludes the proof.

The following result provides a necessary condition on $n$ in order to $f_{k}(n)$ may attain the bound.

Proposition 2.4. Equality in (1) can only hold if $2 n-3=x^{2}$ where $x$ is an integer.

Proof. It follows directly from the second part of Lemma 2.1. 
The result above claims that a skew E-W matrix cannot exist for orders 10, 18, 22, 30, $34,38,46,50,54,58,66,70,74,78,82,90$. The next elementary lemma asserts that a necessary condition for the existence of $n \times n$ skew E-W matrices is that $n-1$ must be a sum of two squares but this is not sufficient. No skew E-W matrix exists for $n=18$ although $n-1=17=4^{2}+1^{2}$. Moreover, there is a conference matrix.

Lemma 2.5. Let $n \equiv 2 \bmod 4$. If $2 n-3=x^{2}$ for some integer $x$ then $n-1=y^{2}+z^{2}$ for some integers $y$ and $z$.

The proof of the Lemma above is based on the observation that $x$ is odd, that is, $x=2 k+1$. As an immediate consequence, it follows that $n-1=k^{2}+(k+1)^{2}$.

Finally, a partial progress toward the solution of Cameron's problem is reported in the following result:

Theorem 2.6. Let $M \in \mathcal{F}_{k}(n)$. Then,

$$
\operatorname{det} M=(2 n-3)(n-3)^{\frac{1}{2} n-1} \Longleftrightarrow \operatorname{det}(M+I)=(2 n-2)(n-2)^{\frac{1}{2} n-1} .
$$

Proof. Let $A^{\prime}=(M+I)(M+I)^{T}$ and $A=M M^{T}$. Observe that since $M$ is skew, $A^{\prime}=A+I$. Since $A^{\prime}$ is positive definite, all of its eigenvalues are positive and their values are $(2 n-2)$ twice and $(n-2), n-2$ times (see [8, p.40]). If $\lambda^{\prime}$ is an eigenvalue of $A^{\prime}$, then $\lambda^{\prime}=\lambda+1$ for an eigenvalue of $A$. The conclusion of the theorem follows.

We recall that $f_{k}(n)$ may attain the bound (1) only when $2 n-3=\alpha^{2}$. For other orders, this question has been tackled in [2] using the cocyclic approach.

\subsection{Finding Skew E-W Matrices}

We now make a sketch of a construction for skew E-W matrices. Skew E-W matrices have been found using this technique [2]. This construction is made up of two steps. Firstly, it consists of looking for $(-1,1)$-matrices with determinant equal to Ehlich-Wotjas' bound, in the case that $2 n-3$ is a square. The search methods usually employed in the literature $[1,23]$ for finding these $(-1,1)$-matrices are based on decomposing $\left[\begin{array}{cc}L^{\prime} & 0 \\ 0 & L^{\prime}\end{array}\right]$ where $L^{\prime}=(n-2) I+2 J$ as the product of a $(-1,1)$-matrix and its transpose. To carry out this first step, we have used the cocyclic approach described in [1], Algorithm 2 p. 869]. Secondly, check if the matrices yielded by step 1 are equivalent to a skew matrix with 1's on the main diagonal. For this second step, we suggest the procedure given in [2], Section 3] where a backtracking search to decide whether or not a $(-1,1)$-matrix $M$ of size $n$ (given as input) is equivalent to a matrix of skew type is performed. If so, a skew-matrix $K$ equivalent to $M$ is provided. Naturally, the search tree is vast and methods to prune it are needed to go beyond the limits of the table produced in [2].

\section{NEW CONSTRUCTION FOR SELF-DUAL CODES}

A linear $[N, K]$ code $C$ over $G F(p)$ is a $K$-dimensional vector subspace of $G F(p)^{N}$, where $G F(p)$ is the Galois field with $p$ elements, $p$ prime. We assume that the reader is familiar with the basic facts of linear codes, and we simply refer to [22] for relevant details in this topics as well as the notation and terminology. 
Previously, self-dual codes have been constructed from some matrices with special structures, such as circulant matrices [11] and weighing matrices [3, 14], in particular Hadamard and conference.

A weighing matrix $W(n, k)$ of order $n$ and weight $k$ is a square $(0,1,-1)$-matrix of size $n$ such that $W \cdot W^{T}=k I_{n}, k \leq n$ (see [13]). Obviously, the determinant of $W(n, k)$ is $k^{n / 2}$. A weighing matrix $W(n, n)$ is a Hadamard matrix, and a weighing matrix $W(n, n-1)$ is called a conference matrix.

Let us point out that

$$
\operatorname{det} W(n, k)<f_{k}(n)<\operatorname{det} W(n, n-1), \quad 1 \leq k \leq n-2, n \geq 4 .
$$

So, if $M+I$ is skew E-W matrix then $M$ is not a weighing matrix.

The following simple but powerful method was introduced in [14] and they have been extensively used in searching for optimal self-dual codes $[3,21]$.

Proposition 3.1. [14] Let $W(n, k)$ be a weighing matrix of order $n$ and weight $k$. Let $\alpha$ be a nonzero element of $G F(p)$ such that

$$
\alpha^{2}+k=0 \bmod p .
$$

Then the matrix

$$
G=[\alpha I, W(n, k)]
$$

generates a self-dual $[2 n, n]$ code $C(n, k)$ over $G F(p)$.

Among weighing matrices, Hadamard and conference matrices provide a large number of self-dual codes with the potential for high minimum distances. We can find a good explanation for this, in the fact that Hadamard and conference matrices provide the maximal determinant among $(-1,1)$-matrices and matrices with 0 's on the main diagonal and \pm 1 's elsewhere, respectively.

When $n \equiv 2 \bmod 4$ no Hadamard matrix exists and conference matrices are symmetric. For small $n$ and $p$, values of $\alpha$ providing self-dual codes with symmetric conference matrices and the distance of these codes were given by Arasu and Gulliver in [3]. Let us point out that for fixed $k=n-1$ the diophantine equation (4) has no solution for some values of $n$ and $p$. For instance, for $p=3$ and $n=14,26$, and 62. Therefore, ternary codes could not be expected to exist from a $n \times n$ conference matrix for these values of $n$.

The lack of solutions in the diophantine equation (4) for some values of $p$ and $n$ when $k=n-1$ motivated the investigation of self-dual codes constructed from skew E-W matrices.

\subsection{Self-Dual Codes from Skew E-W Matrices}

By Theorem 2.3, we can always assume that a skew E-W matrix $M+I$ satisfies the identity (2). In this section, we will use this fact.

In the sequel, a technique for constructing self-dual codes using skew E-W matrices is presented. 
Lemma 3.2. Let $M+I$ be an $n \times n$ skew $E-W$ matrix and $X=\left[\begin{array}{cc}J_{n / 2} & 0 \\ 0 & J_{n / 2}\end{array}\right]$. Then the following identities hold

$$
\text { - } M X=X M, \quad \text { - } X M^{T}+M X=0
$$

Proof. Now let

$$
M=\left[\begin{array}{ll}
B & C \\
D & E
\end{array}\right]
$$

Since $M M^{T}=M^{T} M$, it follows that $M$ and $M M^{T}$ commute and so $L$ commutes with each of $B, C, D$, and $E$, they are square matrices of order $n / 2$. But $L=(n-3) I+2 J$ and so $J$ too commutes with each of $B, C, D$, and $E$. Therefore, we conclude that $M X=X M$ as it was desired. The second identity follows immediately from $M X=X M$.

Proposition 3.3. Let $M+I$ be a skew $E-W$ matrix of order $n$ and suppose that there exist three nonzero elements $\alpha, \beta$, and $\gamma$ of $G F(p)$ such that

$$
\begin{aligned}
\alpha^{2}+\frac{n}{2} \beta^{2}+(n-1) \gamma^{2} & =0 \bmod p, \\
\frac{n}{2} \beta^{2}+2 \gamma^{2} & =0 \bmod p .
\end{aligned}
$$

Then the matrix

$$
G=[\alpha I, \beta X+\gamma M]
$$

generates a self-dual code $C_{\star}(n)$ of length $2 n$ and dimension $n$ over $G F(p)$.

Proof. The statement of this proposition follows by direct inspection from the fact that $G G^{T}=0 \bmod p$ and Lemma 3.2.

An upper bound on the minimum weight of any of these codes is given in the following proposition.

Proposition 3.4. Let $M+I$ be a skew $E-W$ matrix of order $n$. Let $C_{\star}(n)$ be a self-dual code over $G F(p)$ constructed from $M$ using Proposition 3.3. Then

$$
d\left(C_{\star}(n)\right) \leq \begin{cases}3, & n=2 \\ \frac{n}{2}+2, & n \geq 6 .\end{cases}
$$

Proof. Let us take $r_{i}$ and $r_{j}$, two rows of $M$. Both of them chose either from the 1st to $\frac{n}{2}$-th of the rows of $M$ or from $\frac{n}{2}+1$-th to $n$-th. For being $M+I$ a skew E-W matrix then $r_{i} \cdot r_{j}=2$. Therefore $w t\left(r_{i}-r_{j}\right)=\frac{n}{2}$.

Note that the bound for (symmetric) conference matrices is [3]

$$
d(C(n, n-1)) \leq \begin{cases}3, & n=2 \\ \frac{n}{2}+3, & n \geq 6\end{cases}
$$

For some small $n$ and $p$ where a self-dual conference matrix code does not exist for these lengths, values of $\alpha$ and $\beta$ providing self-dual codes from skew E-W matrices and 
TABLE II. Self-dual codes from skew E-W matrices of orders $n=14$ and 26 where a self-dual conference matrix code does not exist for these lengths.

\begin{tabular}{|c|c|c|c|c|c|c|c|c|}
\hline \multirow[b]{2}{*}{$p$} & \multicolumn{4}{|c|}{$\mathrm{N}=28$} & \multicolumn{4}{|c|}{$\mathrm{N}=52$} \\
\hline & $\alpha$ & $\beta$ & $d$ & $d_{b}$ & $\alpha$ & $\beta$ & $d$ & $d_{b}$ \\
\hline 3 & 1 & 1 & 9 & 9 & 1 & 1 & 12 & 15 \\
\hline 5 & 2 & 2 & 8 & $11-12$ & & & & \\
\hline 23 & 9 & 7 & 9 & $11-15$ & & & & \\
\hline
\end{tabular}

the distance of these code have been investigated in the remainder of this subsection and summarized in Table II ( $\gamma$ is assumed to be equal to 1 ). In this table, the column headed $d_{b}$ gives the known bounds $s$ on $d$ for a self-dual code from [10]; if a code attaining this bound is unknown, then the entry $r-s$ indicates that codes with $d=r$ exist and a code with $d \geq r$ is unknown. We have used Magma [5] to compute the distance of the self-dual codes. Actually, we computed the distance of the derived self-dual codes for each possible solution of the diophantine system of Proposition 3.3.

A. [28, 14] Codes

There is only one skew E-W matrix with $n=14$, and this has a form given by

$$
\left[\begin{array}{cccccccccccccc}
1 & 1 & - & - & 1 & - & 1 & 1 & - & - & - & - & - & - \\
- & 1 & 1 & 1 & - & - & 1 & - & - & 1 & - & - & - & - \\
1 & - & 1 & 1 & 1 & - & - & - & - & - & 1 & - & - & - \\
1 & - & - & 1 & - & 1 & 1 & - & - & - & - & - & - & 1 \\
- & 1 & - & 1 & 1 & 1 & - & - & - & - & - & 1 & - & - \\
1 & 1 & 1 & - & - & 1 & - & - & 1 & - & - & - & - & - \\
- & - & 1 & - & 1 & 1 & 1 & - & - & - & - & - & 1 & - \\
- & 1 & 1 & 1 & 1 & 1 & 1 & 1 & 1 & - & 1 & - & - & 1 \\
1 & 1 & 1 & 1 & 1 & - & 1 & - & 1 & - & - & 1 & 1 & 1 \\
1 & - & 1 & 1 & 1 & 1 & 1 & 1 & 1 & 1 & - & 1 & - & - \\
1 & 1 & - & 1 & 1 & 1 & 1 & - & 1 & 1 & 1 & - & 1 & - \\
1 & 1 & 1 & 1 & - & 1 & 1 & 1 & - & - & 1 & 1 & 1 & - \\
1 & 1 & 1 & 1 & 1 & 1 & - & 1 & - & 1 & - & - & 1 & 1 \\
1 & 1 & 1 & - & 1 & 1 & 1 & - & - & 1 & 1 & 1 & - & 1
\end{array}\right] .
$$

The bound for $C_{\star}(14)$ is $d \leq 9$. This is met by self-dual codes $C_{\star}(14)$ with $p=3$ and 23 . For $p=3$, the code $C_{\star}(14)$ is optimal (its distance is the highest possible). For $p=5$ there are two inequivalent codes, both with distance $d=8$. The first with parameters $\alpha=\beta=2$ and $\gamma=1$ and the second with parameters $\alpha=\beta=1$ and $\gamma=2$.

A solution with $\gamma=1$ of the diophantine system for each $p \leq 23$ for which a self-dual code exists is given in Table II.

B. $[52,26]$ Codes

From what we know, there is only one known skew E-W matrix of type with $n=26$, and it is posted on http://personal.us.es/armario/articulos/c26.pdf. The bound for $C_{\star}(26)$ is $d \leq 15$. For $p=3, \alpha=\beta=\gamma=1$ gives $d\left(C_{\star}(26)\right)=12$. 
TABLE III. For $\boldsymbol{n}$ taking all possible orders less than 100 where a skew $\mathrm{E}-\mathrm{W}$ matrix might exist and for $p=3,5$, and 7 , this table provides the pairs $(n, p)$ where the diophantine system (5) has a solution.

\begin{tabular}{ccccccc}
\hline$n$ & 6 & 14 & 26 & 42 & 62 & 86 \\
\hline$p$ & 7 & 3,5 & 3 & & 3,7 & 3 \\
\hline
\end{tabular}

Finally, for all orders less than 100 where a skew E-W matrix might exist and for $p=3,5$, and 7, we have studied (Table III) when the diophantine system (5) has a solution. For $n=42$ there is no solution when $p=3$, 5 , or 7 but there are some for $p=41$.

\section{CONCLUSION}

Here, we have drawn a parallel between skew Hadamard matrices and skew E-W matrices concerning a question about the maximal determinant. Finally, codes constructed from skew E-W matrices are presented.

Summing up, these are the most relevant new results of the paper:

- An upper bound for the determinant of matrices in $\mathcal{F}_{k}(n)$ is provided.

- A problem posted on Cameron's website [7] has been partially solved.

- To the best of our knowledge, it is the first time the method given in Proposition 3.1 has been extended to matrices with pairs of rows and columns that are not orthogonal to each other. An upper bound on the minimum weight of any of these codes is also provided.

As another result, our method provides ternary codes for some values of $N$ where ternary codes could not be expected to exist from a conference matrix. Since some values of $n$ and $p$ exist where the diophantine system (5) has solution but the diophantine equation (4) does not. For instance, for $p=3$ and $n=14,26$, and 62. Although these codes have large distance (see Table II), there is only one optimal ( $p=3$ and $N=28$ ) that appears in [17]. In the other cases, codes with larger distance are known [10]. Nevertheless, for $n=86$ no conference matrix is known. But $2 * 86-3=13^{2}$ and so a skew E-W matrix could exist. If such a matrix for $n=86$ were found then we would have self-dual $[172,86]$-codes for the first time using these techniques. This problem is left open and it is our next goal.

\section{ACKNOWLEDGMENTS}

We would like to express our gratitude to the referees for their many valuable suggestions, which have significantly improved the readability of the paper. The proof of Theorem 2.6, as it is now, was kindly provided by one of the referees. Also, we would like to thank Kristeen Cheng for careful reading of the manuscript. 


\section{REFERENCES}

[1] V. Álvarez, J. A. Armario, M. D. Frau, and F. Gudiel, The maximal determinant of cocyclic $(-1,1)$-matrices over $D_{2 t}$, Linear Algebra Appl 436 (2012), 858-873.

[2] V. Álvarez, J. A. Armario, M. D. Frau, and F. Gudiel, Determinants of $(-1,1)$-matrices of skew-symmetric type: a cocyclic approach, Open Math 13 (2015), 16-25.

[3] K. T. Arasu and T. A. Gulliver, Self-dual codes over $\mathbf{F}_{p}$ and weighing matrices, IEEE Trans Inform Theory 47 (2001), 2051-2055.

[4] J. A. Armario, On $(-1,1)$-matrices of skew type with the maximal determinant and tournaments, In: Algebraic design theory with Hadamard matrices, C. J. Colbourn (Editor), Springer Proceedings in Mathematics and Statistics 133, 2015, pp. 1-11.

[5] W. Bosma and J. J. Cannon, Handbook of Magma functions, 2.9 edition, School of Mathematics and Statistics, University of Sydney, 2002.

[6] F. Bussemaker, I. Kaplansky, B. McKay, and J. Seidel, Determinants of matrices of the conference type, Linear Algebra Appl 261 (1997), 275-292.

[7] P. Cameron, Problem 104 (Peter Cameron's Blog), <http://cameroncounts.wordpress.com /2011/08/19/a-matrix-problem/> 2011, accessed May 26, 2015.

[8] J. H. E. Cohn, On determinants with elements \pm 1 II, Bull London Math Soc 21 (1989), 3642.

[9] H. Ehlich, Determiantenabschätzungen für binäre Matrizen, Math Z 83 (1964), 123-132.

[10] P. Gaborit, Table of self-dual codes http://www.unilim.fr/pages_perso/philippe.gaborit/SD/ SelfDualCodes.htm (2013). Accessed March 26, 2015.

[11] S. D. Georgiou and E. Lappas, Self-dual codes from circulant matrices, Des Codes Cryptrogr 64 (2012), 129-141.

[12] S. Georgiou, C. Koukouvinos, and S. Stylianou, New skew Hadamard matrices and their application in edge desings, Utilitas Math 66 (2004), 121-136.

[13] A. V. Geramita and J. Seberry, Orthogonal Designs: Quadratic Forms and Hadamard Matrices, Marcel Dekker, New York, Basel, 1979.

[14] T. A. Gulliver and M. Harada, New optimal self-dual codes over $G F(7)$, Graphs Combin 15 (1999), 175-186.

[15] J. Hadamard, Résolution d'une question relative aux déterminants, Bull Sci Math 17(2) (1893), 240-246.

[16] J. H. Halton, A combinatorial proof of Cayley's theorem on Pfaffians, J Combin Theory 1(2) (1966), 224-232.

[17] M. Harada, A. Munemasa, and B. Venkov, Classification of ternary extremal self-dual codes of length 28, Math Comput 78(267) (2009), 1787-1796.

[18] K. Horadam, Hadamard Matrices and Their Applications, Princeton University Press, Princenton, NJ, 2007.

[19] Y. Ionin and H. Kharaghani, Balanced generalized weighing matrices and conference matrices, In: The CRC Handbook of Combinatorial Designs, C. J. Colbourn and J. Dinitz (Editors), CRC press, 2nd ed., Boca Raton, 2006 pp. 306-312.

[20] H. Kharaghani and W. Orrick, D-optimal designs, In: The CRC Handbook of Combinatorial Designs, C. J. Colbourn and J. Dinitz (Editors), CRC press, 2nd ed., Boca Raton, 2006, pp. 296-298.

[21] C. Koukouvinos and D. E. Simos, Construction of new self-dual codes over $G F(5)$ using skew-Hadamard matrices, Adv Math Commun 3 (2009), 251-263.

[22] F. J. MacWilliams and N. J. A. Sloane, The Theory of Error-Correcting Codes, North-Holland, Amsterdam, 1977. 
[23] W. Orrick, The maximal $\{-1,1\}$-determinant of order 15, Metrica 62 (2005), 195-219.

[24] W. Orrick, and B. Solomon, The Hadamard Maximal Determinant Problem (website), http://www.indiana.edu/ maxdet/, accessed June 3, 2015

[25] L. Xiao, D. Lin, and F. Bai, Constructing definitive screening designs using conference matrices, J Quality Technol 44 (2012), 1-7.

[26] W. Wojtas, On Hadamard's inequallity for the determinants of order nondivisible by 4, Colloq Math 12 (1964), 73-83. 